\title{
ASYMPTOTIC EXTREMAL GROWTH OF QUASISYMMETRIC FUNCTIONS
}

\section{A. HINKKANEN ${ }^{1)}$}

\section{Introduction}

The purpose of this paper is to determine the asymptotic behaviour of the functions $M_{0}(x, K)$ and $m_{0}(x, K)$, defined below, that describe the maximal and minimal growth of $K$-quasisymmetric functions. The work is based on an earlier paper [5] of the author, which can be regarded as a sequel to the papers $[3,4]$ of W. K. Hayman and the author.

An increasing homeomorphism $f$ of the real axis $\boldsymbol{R}$ onto itself is called $K$-quasisymmetric $(K-q s)$, where $1 \leqq K<\infty$, if

$$
\frac{1}{K} \leqq \frac{f(x+t)-f(x)}{f(x)-f(x-t)} \leqq K
$$

for all $x \in \boldsymbol{R}$ and $t>0$. The function $f$ is quasisymmetric $(q s)$ if it is $K-q s$ for some $K$. The condition (1.1) was formulated by Beurling and Ahlfors [1] who proved that $q s$ functions are precisely the boundary values of those quasiconformal maps of the upper half-plane onto itself that fix the point at infinity.

Some results on the growth of qs functions can be found in Kelingos' paper [6], and a more systematic study has been performed in $[3,4,5]$. Following [4], we set

$$
\begin{aligned}
N_{0}(K)= & \{f \mid f \text { is } \quad K-q s, f(1)=1, f(-1)=-1\}, \\
& M_{0}(x, K)=\max \left\{f(x) \mid f \in N_{0}(K)\right\}, \\
& m_{0}(x, K)=\min \left\{f(x) \mid f \in N_{0}(K)\right\} .
\end{aligned}
$$

We note that by [1], the class $N_{0}(K)$ is compact.

The class $N_{0}(1)$ consists of the identity map only, so that $M_{0}(x, 1)=m_{0}(x, 1)=x$ for all $x$. Let $K$ be fixed, $K>1$. In [5, Theorems 1,2] we constructed piecewise linear odd functions $f$ and $g$ belonging to $N_{0}(K)$, such that $f$ is the largest convex minorant of $M_{0}(x, K)$ and $g$ is the smallest concave majorant of $m_{0}(x, K)$ for $x \geqq-1$. Further

\footnotetext{
1) Research partially supported by the U.S. National Science Foundation.
} 
we found infinitely many points $z_{n}$ and $w_{n}$ tending to $\infty$ as $n \rightarrow \infty$ such that

$$
\begin{aligned}
& f\left(z_{n}\right)=M_{0}\left(z_{n}, K\right), \\
& g\left(w_{n}\right)=m_{0}\left(w_{n}, K\right)
\end{aligned}
$$

for all $n$. It was also shown that if $r=r(K)$ is rational, i.e.

$$
r(K)=\frac{\log K}{\log L}=\frac{p}{q}, \quad L=\frac{1}{2}(K+1),
$$

where $p, q$ are positive relatively prime integers, then the points $z_{n}, w_{n}$ occur at bounded distances. More precisely, we have

by [5, Theorem 4].

$$
\begin{aligned}
z_{n+1}-z_{n} & \leqq 2^{2^{p}+p}, \\
w_{n+1}-w_{n} & \leqq 2^{2^{2 p}+2 p}
\end{aligned}
$$

By $[4$, Theorems 5,6$]$ we have

$$
\begin{gathered}
x^{\alpha_{1}(K)} \leqq M_{0}(x, K) \leqq c_{1}(K) x^{\alpha_{1}(K)}, \\
c_{2}(K) x^{\alpha_{2}(K)} \leqq m_{0}(x, K) \leqq x^{\alpha_{2}(K)}
\end{gathered}
$$

for $x \geqq 1$, where the constants $\alpha_{1}, \alpha_{2}, c_{1}, c_{2}$ depend on $K$ only and can be estimated. Hayman [3, Theorem 1] showed that if $r(K)$ is irrational, then the ratios $M_{0}(x, K) x^{-\alpha_{1}(K)}$ and $m_{0}(x, K) x^{-\alpha_{2}(K)}$ tend to some limits as $x \rightarrow \infty$, say $\gamma_{1}(K)$ and $\gamma_{2}(K)$. He also proved [3, Theorem 5] that if $r(K)$ is rational, then these ratios are asymptotic to some periodic functions of $\log x$ (for example $M_{0}(x, K) \sim x^{\alpha_{1}(K)} \varphi(\log x)$ where $\varphi$ is periodic) but left open the question whether or not $\varphi$ is constant.

In this paper we use the explicit formulas for the above functions $f$ and $g$ together with the fact that $f$ and $M_{0}(x, K)$ as well as $g$ and $m_{0}(x, K)$ have the same asymptotic behaviour, to determine the above functions $\varphi$ and the limits $\gamma_{1}(K)$ and $\gamma_{2}(K)$. This will be done in Sections 4 and 6. In Sections 5 and 7 we study the properties of the functions $\varphi, \gamma_{1}$ and $\gamma_{2}$ to describe the behaviour of $M_{0}$ and $m_{0}$ more precisely. The proofs are based on difference equations arising from the definitions of $f$ and $g$, and these will be considered in Sections 2 and 3. In Section 8 we study the asymptotic oscillation properties of an individual $K-q s$ function. In the final Section 9 we prove a technical result used in Section 6. As stating our results precisely requires some preparation, this will be done in the appropriate sections. 


\section{A difference equation}

If $K>1$, we set $L=L(K)=(1 / 2)(K+1), \Lambda=\Lambda(K)=(1 / 2)\left(1+K^{-1}\right)$,

$$
\begin{aligned}
& r=r(K)=\frac{\log K}{\log L}, \\
& s=s(K)=\frac{\log K^{-1}}{\log \Lambda} .
\end{aligned}
$$

We have $1<r<2, s>2,1 / r+1 / s=1$. If $r \in \mathbf{Q}$, say $r=p / q$ where $p, q$ are positive, relatively prime integers, then $s=p /(p-q), q<p<2 q, 1 \leqq p-q<p$, and $p \geqq 3$, $q \geqq 2$. The numbers $r$ and $s$ are rational or irrational simultaneously.

Consider pairs $(m, n)$ of integers $m \geqq 1, n \geqq 0$. Following [5], we order these pairs so that

$$
K^{m_{1}} L^{n_{1}} \leqq K^{m_{2}} L^{n_{2}} \leqq \ldots
$$

The ordering is unique if and only if $r \notin Q$. If $r \in Q$ and if $K^{m_{k}} L^{n_{k}}$ has the same value for $M \leqq k \leqq N$, we order these pairs $\left(m_{k}, n_{k}\right)$ so that $m_{M}>m_{M+1}>\ldots>m_{N}$.

There is a unique odd piecewise linear continuous function $f$ such that $f(x)=x$ for $0 \leqq x \leqq 1$ and such that the slope of $f$ is $K^{m_{k}} L^{n_{k}}$ on the interval $\left[X_{k-1}, X_{k}\right]$, where

$$
X_{k}-X_{k-1}=2^{n_{k}+1}\left(\begin{array}{c}
m_{k}+n_{k}-1 \\
n_{k}
\end{array}\right) \text { for } k \geqq 1 \text {, and } X_{0}=1 .
$$

It was shown in [5, Theorem 1] that $f \in N_{0}(K)$ and that $f(z)=M_{0}(z, K)$ whenever

$$
z=X_{k-1}+j 2^{n_{k}+1}, \quad 0 \leqq j \leqq\left(\begin{array}{c}
m_{k}+n_{k}-1 \\
n_{k}
\end{array}\right), \quad k \geqq 1 .
$$

Similarly, there is a unique odd function $g$ such that $g(x)=x$ for $0 \leqq x \leqq 1$ and such that the slope of $g$ is $K^{-M_{k}} \Lambda^{N_{k}}$ on $\left[Y_{k-1}, Y_{k}\right]$, where $M_{k} \geqq 1, N_{k} \geqq 0$,

$$
\begin{aligned}
K^{-M_{1}} \Lambda^{N_{1}} \geqq K^{-M_{2}} \Lambda^{N_{2}} \geqq \ldots, \quad Y_{k}-Y_{k-1}=2^{N_{k}+1}\left(\begin{array}{c}
M_{k}+N_{k}-1 \\
N_{k}
\end{array}\right) \text { for } \\
k \geqq 1, \quad \text { and } Y_{0}=1 .
\end{aligned}
$$

By [5, Theorem 1], we have $g \in N_{0}(K)$, and $g(w)=m_{0}(w, K)$ for

$$
w=Y_{k-1}+j 2^{N_{k}+1}, \quad 0 \leqq j \leqq\left(\begin{array}{c}
M_{k}+N_{k}-1 \\
N_{k}
\end{array}\right), \quad k \geqq 1 .
$$

Suppose now that $r(K)$ is rational, say $r(K)=p / q$ as before. Then every slope of $f$ can be written as $K^{m} L^{n}=L^{(m p+n q) / q}$ since $K^{q}=L^{p}$. So the distinct values of the slope of $f$ are given by $L^{m / q}$, where $m$ runs through all positive integers of the form $m=a p+b q$ where $a \geqq 1$ and $b \geqq 0$, and in particular through all integers $m>$ $(p-1) q$. Let the interval of the positive axis where $f$ has the slope $L^{m / q}$ be $\left(x_{m-1}, x_{m}\right)$ 
where $m \geqq 1$ (so $x_{m-1}=x_{m}$ for finitely many small values of $m$ ). We set $A_{n}=$ $x_{n}-x_{n-1}$. By the definition of $f$, we have

where

$$
A_{n}=\sum(a, b) \in F_{n}\left(\begin{array}{c}
a+b-1 \\
b
\end{array}\right) 2^{b+1},
$$

$$
F_{n}=\{(a, b) \mid a \geqq 1, b \geqq 0, a p+b q=n\} .
$$

Clearly $A_{p}=2, A_{p+q}=4$, and $A_{n}=0$ if $1 \leqq n \leqq 2 p-1$ and $p \neq n \neq p+q$. We shall show that

$$
A_{n}=A_{n-p}+2 A_{n-q}, n>p .
$$

If $(a, b) \in F_{n}$, then $(a-1, b) \in F_{n-p}$ if $a \geqq 2$, and $(a, b-1) \in F_{n-q}$ if $b \geqq 1$. Further,

$$
\begin{aligned}
& \left(\begin{array}{c}
a+b-1 \\
b
\end{array}\right) 2^{b+1}=\left(\begin{array}{c}
(a-1)+b-1 \\
b
\end{array}\right) 2^{b+1}+2\left(\begin{array}{c}
a+(b-1)-1 \\
b-1
\end{array}\right) 2^{(b-1)+1} \text {, while } \\
& \left(\begin{array}{c}
(a-1)+b-1 \\
b
\end{array}\right)=0 \text { if } a=1 \text { and }\left(\begin{array}{c}
a+(b-1)-1 \\
b-1
\end{array}\right)=0 \text { if } b=0 .
\end{aligned}
$$

Also if $(a, b) \in F_{n-p}$, then $(a+1, b) \in F_{n}$, and if $(a, b) \in F_{n-q}$, then $(a, b+1) \in F_{n}$. These results imply (2.4).

The equation (2.4) and the values of $A_{n}$ for $1 \leqq n \leqq p$, determine the numbers $A_{n}$ uniquely. By the standard results on difference equations, we can write

$$
A_{n}=\sum_{i=1}^{p} \beta_{i} \lambda_{i}^{n}
$$

for some complex numbers $\beta_{i}$, where the $\lambda_{i}$ are the zeros of the polynomial

$$
P(z)=z^{p}-2 z^{p-q}-1 .
$$

We shall prove that these zeros are simple.

Before studying the polynomial $P$ more closely, we list the corresponding results for the function $g$. Roughly speaking, the role of $q$ is taken by $\mu=p-q \in[1, p / 2)$. The slopes of $g$ are of the form $\Lambda^{n / \mu}$ where $n \geqq p$. If $g$ has the slope $\Lambda^{n / \mu}$ on $\left(y_{n-1}, y_{n}\right)$, and $A_{n}^{\prime}=y_{n}-y_{n-1}$, then

where

$$
A_{n}^{\prime}=\sum_{(a, b) \in F_{n}^{\prime}}\left(\begin{array}{c}
a+b-1 \\
b
\end{array}\right) 2^{b+1}
$$

We have

$$
F_{n}^{\prime}=\{(a, b) \mid a \geqq 1, b \geqq 0, a p+b \mu=n\} \text {. }
$$

$$
A_{n}^{\prime}=A_{n-p}^{\prime}+2 A_{n-\mu}^{\prime} \text {. }
$$

Hence

$$
A_{n}^{\prime}=\sum_{i=1}^{p} \beta_{i}^{\prime}\left(\lambda_{i}^{\prime}\right)^{n}
$$

where the $\lambda_{i}^{\prime}$ are the zeros of

$$
Q(z)=z^{p}-2 z^{p-\mu}-1=z^{p}-2 z^{q}-1,
$$

all of them simple zeros. 


\section{On the polynomials $P$ and $Q$}

We study the polynomial

$$
P(z)=z^{p}-2 z^{m}-1,
$$

where $p$ and $m$ are relatively prime (in particular, $p$ and $m$ cannot both be even) and $1 \leqq m<p$. We shall apply the results to $m=p-q$ and to $m=q$.

Lemma 1. The polynomial $P$ has $p$ simple zeros $\lambda_{1}, \ldots, \lambda_{p}$. One of them, say $\lambda_{1}$, is the unique positive zero of $P$ and

$$
\left|\lambda_{i}\right|<\lambda_{1}, \quad 2 \leqq i \leqq p .
$$

Further, $1<\lambda_{1}<3, \lambda_{1}^{p-m}<3$, and $\lambda_{1}<\sqrt{3}$ if $p-m \geqq 2$, while $2<\lambda_{1}<2+2^{1-p}$ if $m=p-1$.

We have

$$
P^{\prime}(z)=p z^{p-1}-2 m z^{m-1}=0
$$

if $z=0$ (which is not a zero of $P$ ) or if $z^{p-m}=2 m / p$. Hence if $P(z)=P^{\prime}(z)=0$ (so $z \neq 0$ ), we have $z^{m}=-(2(1-m / p))^{-1}$ and $z^{p}=-m /(p-m)$. This implies that with $\varrho=m / p \in(0,1)$, we have

$$
2 \varrho^{\varrho}(1-\varrho)^{1-\varrho}=1 .
$$

This is satisfied only if $\varrho=1 / 2$, i.e. $p=2 m$, which is against our assumption. Hence all the zeros of $P$ are simple.

We have $P(0)=-1<0$. For real $z, P(z)$ is real, and for $z>0$, we have $P^{\prime}(z)<0$ for $0<z<(2 m / p)^{1 /(p-m)}=A_{0}$ and $P^{\prime}(z)>0$ for $z>A_{0}$. Hence $P$ has a unique positive zero $\lambda_{1}$. We have $\lambda_{1}>1$ since $P(1)=-2<0$. If $\lambda_{1}^{p} \geqq 3 \lambda_{1}^{m}$, then $P\left(\lambda_{1}\right) \geqq \lambda_{1}^{m}-1>0$. Hence $\lambda_{1} \leqq \lambda_{1}^{p-m}<3$, and consequently $\lambda_{1}<\sqrt{3}$ if $p-m \geqq 2$. If $m=p-1$, then $P(2)=(2-2) 2^{p-1}-1<0$, so that $\lambda_{1}>2$, while $P\left(2+2^{1-p}\right)>2^{1-p} 2^{p-1}-1=0$, so that $\lambda_{1}<2+2^{1-p}$.

Suppose that $2 \leqq i \leqq p$. Then $\left|\lambda_{i}\right|^{p}=\left|2 \lambda_{i}^{m}+1\right|<2\left|\lambda_{i}\right|^{m}+1$ unless $\lambda_{i}^{m}>0$. So $P\left(\left|\lambda_{i}\right|\right)<0$ and hence $\left|\lambda_{i}\right|<\lambda_{1}$. If $\left|\lambda_{i}\right| \leqq 1$, then $\left|\lambda_{i}\right|<\lambda_{1}$. Suppose then that $\left|\lambda_{i}\right|>1$ and that $\lambda_{i}^{m}>0$. Then $\lambda_{i}^{p}=2 \lambda_{i}^{m}+1>0$ and $(2 \pi)^{-1}$ arg $\lambda_{i}=k / m=l / p$ for some integers $k, l$ with $0 \leqq k<m, 0 \leqq l<p$. Since $k p=l m$, we have $m \mid k$, so $k / m=0$ and $\lambda_{i}>0$. But then $\lambda_{i}=\lambda_{1}$, which is impossible. Hence $\left|\lambda_{i}\right|<\lambda_{1}$ for $2 \leqq i \leqq p$. Lemma 1 is proved.

3.1. We make a few remarks concerning the case $m=p-q$, where $q<p<2 q$.

Remark 1. A more careful analysis shows that for $2 \leqq i \leqq p$, we have

$$
\left|\lambda_{i}\right| \leqq \lambda_{1}\left(1-A p^{-3}\right),
$$

where $A$ is a positive absolute constant. This seems to be best possible apart from the best value of $A$. 
Remark 2. Applying Rouche's theorem to $z^{p}$ and $2 z^{m}+1$ on the unit circle we see that $P$ has $m$ zeros in the unit disk. The other $p-m$ zeros lie in $\{|z|>1\}$, except if $p$ and $m$ are odd, in which case $P(-1)=0$. Also all zeros $z_{0}$ of $P$ satisfy $\left|z_{0}\right| \geqq B$, where $B \in(0,1)$ is the unique positive zero of $z^{p}+2 z^{m}-1$.

Remark 3. In Section 5 we will consider what happens to various quantities as $K_{n} \rightarrow K, r\left(K_{n}\right)=p_{n} / q_{n}, r\left(K_{n}\right) \rightarrow r \in(1,2)$, where $r$ is irrational. It might be of interest to study what happens to the zeros of

$$
P_{n}(z)=z^{p}-2 z^{m}-1, \quad p=p_{n}, \quad m=p_{n}-q_{n}
$$

as $n \rightarrow \infty$. It seems plausible that for a portion $1 / r$ of the zeros (whose number $\rightarrow \infty$ ), the $q_{n}$-th powers of the zeros cluster towards the circle $\{|z|=C\}$, where $C>1$ is the unique positive zero of $x^{r}-2 x^{r-1}-1$, while for a portion $1-r^{-1}$ of the zeros, the $q_{n}$-th powers cluster towards the circle $\{|z|=B\}$, where $0<B<1$ and $B$ is the unique positive zero of $x^{r}+2 x^{r-1}-1$. However, any useful information would have to be more precise.

3.2. Now we can determine the numbers $\beta_{i}$ in (2.5) and $\beta_{i}^{\prime}$ in (2.8).

Lemma 2. Let $\lambda_{i}, 1 \leqq i \leqq p$, be the zeros of $P$ given by (3.1) with $m=p-q$. Then

$$
\beta_{i}=\frac{2}{\lambda_{i} P^{\prime}\left(\lambda_{i}\right)}=\frac{2}{p+2 q \lambda_{i}^{p-q}} \neq 0
$$

in (2.5). Let $\lambda_{i}^{\prime}, 1 \leqq i \leqq p$, be the zeros of P given by (3.1) with $m=q$. Then

$$
\beta_{i}^{\prime}=\frac{2}{\lambda_{i}^{\prime} P^{\prime}\left(\lambda_{i}^{\prime}\right)}=\frac{2}{p+2(p-q) \lambda_{i}^{\prime q}} \neq 0
$$

$\operatorname{in}(2.8)$.

It suffices to prove that with $\beta_{i}$ given by (3.2), (2.5) is true for $1 \leqq n \leqq p$. Recall that $A_{p}=2$ and $A_{n}=0$ for $1 \leqq n<p$. Let $R$ be so large that the disk $\{|z|<R\}$ contains all zeros of $P$. Then the residue theorem gives

$$
\frac{1}{2 \pi i} \int_{|z|=R} \frac{z^{n} d z}{z P(z)}=\sum_{i=1}^{p} \frac{\lambda_{i}^{n}}{\lambda_{i} P^{\prime}\left(\lambda_{i}\right)}, \quad n \geqq 1,
$$

while for all large $R$ we also have, with $z=R e^{i \theta}$,

$$
\begin{aligned}
& \frac{1}{2 \pi i} \int_{|z|=R} \frac{z^{n-1} d z}{P(z)}=\frac{1}{2 \pi} \int_{0}^{2 \pi} \frac{z^{n-p} d \theta}{1-\left(2 z^{m}+1\right) z^{-p}} \\
= & \frac{1}{2 \pi} \int_{0}^{2 \pi} d \theta z^{n-p} \sum_{k=0}^{\infty}\left(2 z^{m-p}+z^{-p}\right)^{k} \\
= & \frac{1}{2 \pi} \int_{0}^{2 \pi} d \theta z^{n-p}\left(1+2 z^{m-p}+z^{-p}+\sum_{k=p+1}^{\infty} a_{k} z^{-k}\right)
\end{aligned}
$$


for some numbers $a_{k}$. Hence this integral is equal to 1 if $n=p$, and equal to zero if $1 \leqq n<p$. This proves the claim concerning the $\beta_{i}$, and for the $\beta_{i}^{\prime}$ the proof is the same. It is routine to verify that the second equality holds in (3.2) and (3.3). Lemma 2 is proved.

Remark. Since $\left|\lambda_{i}\right|^{p-q} \leqq \lambda_{1}^{q}=\lambda_{1}^{p-m}<3$, we have $\left|p+2 q \lambda_{1}^{p-q}\right|<p+6 q<7 p$, hence $\left|\beta_{i}\right|>2 /(7 p)$. Since $\lambda_{1}>1$, we have $\beta_{1}<1 / p$. One can show that $\left|\beta_{i}\right| \leqq A_{1}(r) / p$, where $A_{1}(r)$ depends only on the ratio $r=p / q, A_{1}(r)$ remains bounded as $r \rightarrow 1$ (i.e. as $K \rightarrow \infty$ ), but $A_{1}(r)$ might tend to $\infty$ as $r \rightarrow 2$ (i.e. as $K \rightarrow 1$ ).

Further, one can show that $0<A_{2}(r) \leqq p\left|\beta_{i}^{\prime}\right| \leqq A_{3}(r)$, where the function $A_{2}(r) \rightarrow$ $\infty$ only when $r \rightarrow 1$ and $A_{3}(r) \rightarrow \infty$ only when $r \rightarrow 2$.

\section{Asymptotic behaviour of $M_{0}$ and $m_{0}$ for rational $r(K)$}

Let $r(K)$ be rational, say $r(K)=p / q$, where $p, q$ are positive and coprime. As mentioned in Section 1, the asymptotic behaviour of $M_{0}(x, K)$ and $m_{0}(x, K)$ is the same as that of $f$ and $g$, respectively. The piecewise linear functions $f$ and $g$ are determined by their slopes $L^{n / q}$ and $\Lambda^{n / \mu}$, where $\mu=p-q$, and by the numbers $A_{n}, A_{n}^{\prime}$, which by Lemmas 1 and 2 are asymptotically given by $\beta_{1} \lambda_{1}^{n}$ and $\beta_{1}^{\prime}\left(\lambda_{1}^{\prime}\right)^{n}$. This allows us to determine the asymptotic behaviour of $M_{0}$ and $m_{0}$. Furthermore, we shall show how $\beta_{1}, \lambda_{1}, \beta_{1}^{\prime}, \lambda_{1}^{\prime}$ are connested to quantities studied in [4].

Let us recall [4, Theorems 5,6$]$ that if $K>1$, then we have

$$
\begin{gathered}
x^{\alpha_{1}} \leqq M_{0}(x, K) \leqq c_{1}(K) x^{\alpha_{1}}, \\
c_{2}(K) x^{\alpha_{2}} \leqq m_{0}(x, K) \leqq x^{\alpha_{2}}
\end{gathered}
$$

for $x \geqq 1$, where $\alpha_{1}=\alpha_{1}(K)$ and $\alpha_{2}=\alpha_{2}(K)$ are obtained as follows (see [1] or [4, Lemma 1]).

Lemma A. If $\alpha>0$, then the function

$$
g_{\alpha}(x)=|x|^{\alpha} \operatorname{sign} x
$$

is $K_{\alpha}-q s$, where the best possible $K_{\alpha}$ is determined as follows. Let $t_{\alpha}$ be the solution of

$$
(t+1)^{1-\alpha}+(t-1)^{1-\alpha}=2,
$$

so that $1<t_{\alpha}<2$. Further set

$$
\begin{aligned}
q_{\alpha} & =\left[\left(t_{\alpha}+1\right)^{\alpha}-1\right]\left[\left(t_{\alpha}-1\right)^{\alpha}+1\right]^{-1} \\
& =\left[\left(t_{\alpha}+1\right) /\left(t_{\alpha}-1\right)\right]^{\alpha-1}=2\left(t_{\alpha}+1\right)^{\alpha-1}-1 .
\end{aligned}
$$

Then $K_{\alpha}=q_{\alpha}$ for $\alpha>1, K_{\alpha}=1 / q_{\alpha}$ for $0<\alpha<1$, and $K_{1}=1$.

The quantity $q_{\alpha}$ is a continuous strictly increasing function of $\alpha$. Hence for any given $K>1$, there are unique numbers $\alpha_{1}(K)>1$ and $\alpha_{2}(K) \in(0,1)$ such that $K=$ $K_{\alpha}$ for $\alpha=\alpha_{1}(K)$ and for $\alpha=\alpha_{2}(K)$. 
We define $D_{0}=\left(\lambda_{1}-1\right) \beta_{1}^{-1}$,

$$
D_{1}=\beta_{1} L_{0}^{\alpha_{1}}\left\{\left(\lambda_{1}^{\alpha_{1}}-1\right)^{-1}-\left(\lambda_{1}-1\right)^{-1}\right\}<0,
$$

$D_{2}=D_{0}^{\alpha_{1}-1}$, and we define $D_{0}^{\prime}, D_{1}^{\prime}, D_{2}^{\prime}$, by the same formulas, replacing $\beta_{1}, \lambda_{1}, \alpha_{1}$ by $\beta_{1}^{\prime}, \lambda_{1}^{\prime}, \alpha_{2}$. We prove the following result.

Theorem 1. Let $r(K)$ be rational. Then as $x \rightarrow \infty$, we have

$$
\begin{aligned}
& M_{0}(x, K) x^{-\alpha_{1}} \sim \varphi_{1}\left(\log x+\log D_{0}\right), \\
& m_{0}(x, K) x^{-\alpha_{2}} \sim \varphi_{2}\left(\log x+\log D_{0}^{\prime},\right.
\end{aligned}
$$

where $\varphi_{1}$ and $\varphi_{2}$ are continuous periodic functions, $\varphi_{1}$ has period $\log \lambda_{1}, \varphi_{2}$ has period $\log \lambda_{1}^{\prime}$, and

$$
\begin{array}{ll}
\varphi_{1}(v)=D_{1} \exp \left(-v \alpha_{1}\right)+D_{2} \exp \left(v\left(1-\alpha_{1}\right)\right), & 0 \leqq v<\log \lambda_{1}, \\
\varphi_{2}(v)=D_{1}^{\prime} \exp \left(-v \alpha_{2}\right)+D_{2}^{\prime} \exp \left(v\left(1-\alpha_{2}\right)\right), & 0 \leqq v<\log \lambda_{1}^{\prime} .
\end{array}
$$

Furthermore,

$$
\begin{gathered}
\log \lambda_{1}=(\log L)(q(\alpha-1))^{-1}=q^{-1} \log \left(t_{\alpha}+1\right), \quad \alpha=\alpha_{1}(K), \\
\beta_{1}=2 q^{-1}\left(r+2\left(t_{\alpha}+1\right)^{r-1}\right)^{-1}, \quad \alpha=\alpha_{1}(K), \\
\log \lambda_{1}^{\prime}=(\log 1 / \Lambda)((p-q)(1-\alpha))^{-1}=(p-q)^{-1} \log \left(t_{\alpha}+1\right), \quad \alpha=\alpha_{2}(K), \\
\beta_{1}^{\prime}=2(p-q)^{-1}\left(s+2\left(t_{\alpha}+1\right)^{s-1}\right)^{-1}, \quad \alpha=\alpha_{2}(K), \quad s=s(K) .
\end{gathered}
$$

4.1. We prove (4.3), (4.5), (4.7) and (4.8). The proof of (4.4), (4.6), (4.9) and (4.10) is similar.

Clearly (4.8) follows from (3.2) and (4.7). Next let $P$ be given by (3.1) with $m=p-q$. By Lemma $1, \lambda_{1}$ is the unique positive zero of $P$. So to prove (4.7), it suffices to show that $P(\delta)=0$, where $\delta=\left(t_{\alpha}+1\right)^{1 / q}$. By Lemma $A$ and (4.2) we have $K=$ $[(t+1) /(t-1)]^{\alpha_{1}-1}$, where $t=t_{\alpha}, \alpha=\alpha_{1}(K)$, and $L=(t+1)^{\alpha-1}$, which proves the second equality in (4.7). We get

$$
\begin{aligned}
P(\delta) & =\delta^{p}\left(1-2 \delta^{-q}\right)-1=\left(\delta^{q}\right)^{r-1}\left(\delta^{q}-2\right)-1 \\
& =\left(t_{\alpha}+1\right)^{r}\left(t_{\alpha}-1\right)\left(t_{\alpha}+1\right)^{-1}-1 \\
& =L^{r /(\alpha-1)} K^{-1 /(\alpha-1)}-1=0
\end{aligned}
$$

since $L^{r}=K$ by the definition of $r$. This proves (4.7).

4.2. It remains to consider the asymptotic behaviour of $M_{0}(x, K)$. By (2.5) and Lemmas 1 and 2 we have

$$
A_{n}=\beta_{1} \lambda_{1}^{n}\left(1+\sum_{i=2}^{p}\left(\beta_{i} / \beta_{1}\right)\left(\lambda_{i} / \lambda_{1}\right)^{n}\right)=\beta_{1} \lambda_{1}^{n}\left(1+E_{n}\right),
$$

where $\left|E_{n}\right| \leqq E \sigma^{n}$ for some $\sigma, 0<\sigma<1$, and some positive $E$. The function $f$ has the slope $L^{n / q}$ on $\left(x_{n-1}, x_{n}\right)$, and

$$
x_{n}=1+\sum_{i=1}^{n} A_{i} .
$$


Suppose that $x_{n} \leqq x<x_{n+1}$. Then

$$
f(x)=1+\sum_{i=1}^{n} A_{i} L^{i / q}+\left(x-x_{n}\right) L^{(n+1) / q} .
$$

By [5, Theorem 1] we have $f\left(x_{n}\right)=M_{0}\left(x_{n}, K\right)$. By [5, Theorem 4], there are points $z_{1}, z_{2}$ such that $x_{n} \leqq z_{1} \leqq x \leqq z_{2} \leqq x_{n+1}, z_{2}-z_{1} \leqq 2^{2^{p}+p}$, and $f\left(z_{i}\right)=M_{0}\left(z_{i}, K\right)$ for $i=1$, 2. Since $f$ and $M_{0}(x, K)$ are increasing and $f \leqq M_{0}$ (since $f \in N_{0}(K)$ ), we have

$$
0 \leqq M_{0}(x, K)-f(x) \leqq f\left(z_{2}\right)-f(x)=\left(z_{2}-x\right) L^{(n+1) / q} \leqq B L^{n / q},
$$

where $B=L^{1 / q} 2^{2^{p}+p}$.

Next we estimate $x_{n}$ and $f\left(x_{n}\right)$. We have

$$
x_{n}=1+\sum_{l=1}^{n} \beta_{1} \lambda_{1}^{i}\left(1+E_{i}\right)=\beta_{1} \lambda_{1}^{n+1}\left(\lambda_{1}-1\right)^{-1}\left(1+S_{n}\right)
$$

where $\left|S_{n}\right| \leqq S \sigma^{n}$ for some positive $S$. Further, we have

$$
f\left(x_{n}\right)=1+\sum_{i=1}^{n} \beta_{1}\left(\lambda_{1} L^{1 / q}\right)^{i}\left(1+E_{i}\right)=\beta_{1} \lambda_{1}^{\alpha(n+1)}\left(\lambda_{1}^{\alpha}-1\right)\left(1+T_{n}\right)
$$

where $\alpha=\alpha_{1}(K)$ and $\left|T_{n}\right| \leqq T \sigma^{n}$ for some positive $T$. Note that $\lambda_{1} L^{1 / q}=\lambda_{1}^{\alpha}$ by (4.7).

We write $x=x_{n} e^{v}$ and deduce from (4.11), (4.12), (4.13) and (4.14) that

$$
\begin{array}{r}
M_{0}(x, K) x^{-\alpha}=\beta_{1}^{1-\alpha}\left(\lambda_{1}-1\right)^{-\alpha} e^{-v \alpha}\left(1+S_{n}\right)^{-\alpha} \\
\cdot\left\{\left(\lambda_{1}^{\alpha}-1\right)^{-1}\left(1+T_{n}\right)+\left(e^{v}-1\right)\left(\lambda_{1}-1\right)^{-1}\left(1+S_{n}\right)\right\},
\end{array}
$$

where we have included the effect of $M_{0}(x, K)-f(x)$ in the $T_{n}$-term, as we may, and divided through by $\lambda_{1}^{\alpha(n+1)}$. As $x \rightarrow \infty$, we have $n \rightarrow \infty$ and so $S_{n}, T_{n} \rightarrow 0$. We have $0 \leqq v \leqq \log \left(x_{n+1} / x_{n}\right)=\log \lambda_{1}+o(1)$ and

$$
\begin{aligned}
v=\log x-\log x_{n} & =\log x+\log D_{0}+(n+1) \log \lambda_{1}+\log \left(1+S_{n}\right) \\
& \equiv \log x+\log D_{0}+o(1)\left(\bmod \log \lambda_{1}\right) .
\end{aligned}
$$

In view of the definition (4.5), (4.15) now implies (4.3).

We claimed that $\varphi_{1}$ is continuous, i.e. $\varphi_{1}(0)=\lim \varphi_{1}(v)$ as $v \rightarrow \log \lambda_{1}-$, which reads

$$
D_{1}+D_{2}=D_{1} \lambda_{1}^{-\alpha}+D_{2} \lambda_{1}^{1-\alpha} \text {, }
$$

i.e. $\left(\lambda_{1}^{\alpha}-1\right) D_{1}+\left(\lambda_{1}^{\alpha}-\lambda_{1}\right) D_{2}=0$. This follows straight from the definitions of $D_{1}$ and $D_{2}$. Theorem 1 is proved.

\section{On the functions $\varphi_{1}(v)$ and $\varphi_{2}(v)$}

In this section we study the functions $\varphi_{i}(v)=\varphi_{i}(v, K)$ for $i=1,2$, given by (4.5) and (4.6). This will show more precisely how much $M_{0}(x, K) x^{-\alpha_{1}(K)}$ and $m_{0}(x, K) x^{-\alpha_{2}(K)}$ can oscillate.

First we consider $\varphi_{1}(v)$. We may assume that $0 \leqq v \leqq \log \lambda_{1}$. We have $e^{v \alpha_{1}} \varphi^{\prime}(v)=-\alpha_{1} D_{1}-\left(\alpha_{1}-1\right) D_{2} e^{v}$ which vanishes at only one point $v_{0}$. Since $\varphi_{1}$ is not 
constant and $\varphi_{1}(0)=\varphi_{1}\left(\log \lambda_{1}\right)$, we have $0<v_{0}<\log \lambda_{1}$. Since $\varphi_{1}^{\prime}(0+)>0$, as one can check, $\varphi_{1}$ takes its maximum value at $v=v_{0}$ and its minimum value at $v=0$. We write

so that

$$
\varrho=\varrho(K)=\frac{\max \varphi_{1}}{\min \varphi_{1}}=\frac{\varphi_{1}\left(v_{0}\right)}{\varphi_{1}(0)}>1,
$$

$$
\varrho(K)=\frac{(\alpha-1)^{\alpha-1}}{\alpha^{\alpha}} \frac{\lambda^{\alpha}-1}{\lambda-1}\left(1-\frac{\lambda-1}{\lambda^{\alpha}-1}\right)^{1-\alpha},
$$

where $\alpha=\alpha_{1}(K)$ and $\lambda=\lambda_{1}$. If $r(K)$ is irrational, we set $\varrho(K)=1$. We prove the following result, which shows that $\varrho(K)$ is bounded, that $\varrho(K)$ is continuous at $K=K_{0}$ if $r\left(K_{0}\right)$ is irrational and that $\varrho(K)$ does not tend to a limit as $K \rightarrow \infty$.

Theorem 2. We have $\varrho(K)<((3+\sqrt{5}) / 2)^{1 / 3}<1.38$ for all $K$. If $K_{i} \rightarrow K$ and $r=r(K)$ is irrational, then $\varrho\left(K_{i}\right) \rightarrow 1$, and

$$
\varphi_{1}\left(0, K_{i}\right) \rightarrow \gamma_{1}(K)=\alpha^{-1} 2^{1-\alpha}[\log (t+1)]^{\alpha-1}\left[r+2(t+1)^{r-1}\right]^{\alpha-1},
$$

where $t=t_{\alpha}$ and $\alpha=\alpha_{1}(K)$. If $K_{i} \rightarrow 1$, then $\varrho\left(K_{i}\right) \rightarrow 1$. If $K_{i} \rightarrow \infty$ and $r\left(K_{i}\right)=$ $p_{i} / q_{i}$, then

$$
\begin{gathered}
\limsup _{i \rightarrow \infty} \varrho\left(K_{i}\right)=e^{-1} \sigma_{1} / \log \sigma_{1} \leqq 2 /(e \log 2)<1.0615, \\
\liminf _{i \rightarrow \infty} \varrho\left(K_{i}\right)=e^{-1} \sigma_{2} / \log \sigma_{2},
\end{gathered}
$$

where

$$
\begin{aligned}
\sigma_{i}=2^{1 / \tau_{i}}, & \tau_{i}=M_{i}\left(1-2^{-1 / M_{i}}\right), \quad i=1,2, \\
M_{1} & =\liminf _{i \rightarrow \infty} p_{i}-q_{i}, \\
M_{2} & =\limsup _{i \rightarrow \infty} p_{i}-q_{i} .
\end{aligned}
$$

If $M_{i}=\infty$, then $\tau_{i}=1 / \log 2, \sigma_{i}=e$, and $e^{-1} \sigma_{i} / \log \sigma_{i}=1$. Otherwise $e^{-1} \sigma_{i} / \log \sigma_{i}>1$. We have $M_{i} \geqq 1$. Thus $\sigma_{i} \leqq 4$ and $e^{-1} \sigma_{i} / \log \sigma_{i} \leqq 2 /(e \log 2)$ since $M\left(1-2^{-1 / M}\right)$ increases from $1 / 2$ to $\log 2$ as $M$ increases from 1 to $\infty$. The theorem shows that even for large $K$, it is possible to have $\varrho(K)$ bounded away from 1 . The upper bound $((3+\sqrt{5}) / 2)^{1 / 3}$ for $\varrho(K)$ is not best possible, and it may be that $\varrho(K)<$ $2 /(e \log 2)$ for all $K$. In Theorem 5, Section 6 , the function $\gamma_{1}(K)$ will be identified as the limit of $M_{0}(x, K) x^{-\alpha_{1}(K)}$ as $x \rightarrow \infty$ when $r(K)$ is irrational.

Hayman [3, proof of Theorem 5] showed that $\varrho(K) \leqq \lambda_{1}^{\alpha_{1}(K)}$, which also remains below an absolute constant, for example $2 \sqrt{3}$.

5.1. To prove Theorem 2, suppose that $r(K)=p / q$ and note that

$$
\varphi_{1}(0, K)=D_{1}+D_{2}=\left(\frac{\lambda_{1}-1}{\beta_{1}}\right)^{\alpha_{1}-1} \frac{\lambda_{1}-1}{\lambda_{1}^{\alpha_{1}}-1} .
$$


Further, by (4.7), $\lambda_{1}=(t+1)^{1 / q}$ where $t=t_{\alpha}$ and $\alpha=\alpha_{1}(K)$, and by Lemma 2 we have $2 \beta_{1}^{-1}=p+2 q(t+1)^{r-1}$. Hence if we take here $K=K_{i} \rightarrow K_{0}$ where $p=p_{i}, q=q_{i} \rightarrow \infty$ and $p_{i} / q_{i} \rightarrow r\left(K_{0}\right)$, we get (5.2), since $\alpha_{1}(K)$ and $t_{\alpha}$ are continuous functions of $K$.

If $K_{i} \rightarrow K$ and $r(K)$ is irrational, then $q_{i} \rightarrow \infty, \lambda_{1}-1 \sim q_{i}^{-1} \log (t+1)$ and $\lambda_{1}^{\alpha}-1 \sim q_{i}^{-1} \alpha \log (t+1)$. Hence an analysis of (5.1) shows that $\varrho\left(K_{i}\right) \rightarrow 1$.

Similarly we see that $\varrho\left(K_{i}\right) \rightarrow 1$ if $K_{i} \rightarrow 1$.

Suppose next that $K_{i} \rightarrow \infty$ in such a way that $p_{i}-q_{i}=m$ is a constant. Then by [4, Theorem 6] we have

and

$$
r\left(K_{i}\right)=\frac{\log K_{i}}{\log L_{i}}=\frac{q_{i}+m}{q_{i}}
$$

$$
q_{i}=m \frac{\log K_{i}}{r\left(K_{i}\right) \log \left(K_{i} / L_{i}\right)} \sim \frac{m \alpha \log 3}{\log 2}, \quad \alpha=\alpha_{1}\left(K_{i}\right) .
$$

Hence $\lambda^{\alpha}=\left(t_{\alpha}+1\right)^{\alpha / a_{i} \rightarrow 2^{1 / m}}$ since $t_{\alpha} \rightarrow 2$. Further $\lambda-1 \sim(\log 2) /(m \alpha)$. It follows that

Since

$$
\left(1-\frac{\lambda-1}{\lambda^{\alpha}-1}\right)^{1-\alpha} \rightarrow 2^{1 / \tau}, \quad \tau=m\left(2^{1 / m}-1\right) .
$$

$$
\frac{(\alpha-1)^{\alpha-1}}{\alpha^{\alpha}} \frac{\lambda^{\alpha}-1}{\lambda-1} \rightarrow e^{-1} \tau / \log 2,
$$

it follows from (5.1) that $\varrho\left(K_{i}\right) \rightarrow e^{-1} \sigma / \log \sigma$, where $\sigma=2^{1 / \tau}$. Now (5.3) and (5.4) follow from this result.

5.2. It remains to find an upper bound for $\varrho(K)$. Suppose that $r(K)=p / q$ and that $1<\alpha<2$. Note that $q=2$ only if $r(K)=3 / 2$, i.e. if $\alpha_{1}(K)=2$. Now $\alpha<$ $\left(C^{\alpha}-1\right) /(C-1)<\alpha C$ for any $C>1$. Hence

$$
\begin{aligned}
\varrho(K) & =\left(1-\frac{1}{\alpha}\right)^{\alpha} /\left\{\left(1-\frac{\lambda-1}{\lambda^{\alpha}-1}\right)^{\alpha-1}(\alpha-1) \frac{\lambda-1}{\lambda^{\alpha}-1}\right\} \\
& <\frac{\lambda^{\alpha}-1}{\alpha(\lambda-1)}<\lambda=(t+1)^{1 / q} \leqq(t+1)^{1 / 3} .
\end{aligned}
$$

Since $t_{\alpha}$ is an increasing function of $\alpha$ and $t_{\alpha}=(1+\sqrt{5}) / 2$ when $\alpha=2$, we have $\varrho(K)<((3+\sqrt{5}) / 2)^{1 / 3}<1.38$ for $1<\alpha_{1}(K)<2$.

If $\alpha \geqq 2$, we could use the inequality $\alpha<\left(C^{\alpha}-1\right) /(C-1)<\alpha C^{\alpha-1}$ valid for $\alpha>1$, $C>1$, to get $\varrho(K)<\lambda^{\alpha-1}=L^{1 / q}$. Since further $1 / q \leqq(p-q) / q=\log (K / L) / \log L$ and $K \leqq 2 L$, this gives $\varrho(K)<2$ for all $\alpha>1$. We get a better upper bound by observing that for any fixed $\alpha \geqq 2$, the right hand side of (5.1) is an increasing function of $\lambda$ for $\lambda>1$. Hence using the bound $\lambda \leqq 2^{1 /(\alpha-1)}$ obtained above we get

$$
\varrho(K)<\frac{(\alpha-1)^{\alpha-1}}{2 \alpha^{\alpha}} \frac{\left(2^{\alpha /(\alpha-1)}-1\right)^{\alpha}}{2^{1 /(\alpha-1)}-1}=V(\alpha),
$$


say. When $\alpha$ increases from 2 to $\infty, V(\alpha)$ decreases from $9 / 8$ to $2 /(e \log 2)$, so that $\varrho(K)<9 / 8=1.125$ for $\alpha \geqq 2$. This proves Theorem 2 .

We remark that if $\alpha=2$, then $K=2+\sqrt{5}, r(K)=3 / 2$, and $\varrho(K)=(2+\sqrt{5}) / 4<$ 1.05902 .

5.3. One can obtain similar results for the function $\varphi_{2}(v, K)$. There is a unique point $v_{0} \in\left(0, \log \lambda_{1}^{\prime}\right)$ such that $\varphi_{2}^{\prime}\left(v_{0}\right)=0$. The function $\varphi_{2}$ takes its maximum at $v=0$ and its minimum at $v=v_{0}$. We set

$$
\tilde{\varrho}(K)=\frac{\max \varphi_{2}}{\min \varphi_{2}}=(1-\alpha)^{1-\alpha} \alpha^{\alpha}\left(\frac{\lambda-1}{\lambda^{\alpha}-1}\right)^{\alpha}\left(1-\frac{\lambda^{\alpha}-1}{\lambda-1}\right)^{\alpha-1},
$$

where $\alpha=\alpha_{2}(K)$ and $\lambda=\lambda_{1}^{\prime}$. If $r(K)$ is irrational, we set $\varrho(K)=1$. The following result is proved in the same way as Theorem 2 .

Theorem 3. We have $\varrho(K)<4$ for all $K$. If $K_{i} \rightarrow 1$ or $K_{i} \rightarrow \infty$, we have $\varrho\left(K_{i}\right)$ $\rightarrow 1$. If $K_{i} \rightarrow K$ and $r(K)$ is irrational, we hare $\tilde{\varrho}\left(K_{i}\right) \rightarrow 1$ and $\varphi_{2}\left(0, K_{i}\right) \rightarrow \gamma_{2}(K)=$ $\alpha^{-1} 2^{1-\alpha}[\log (t+1)]^{\alpha-1}\left[s+2(t+1)^{s-1}\right]^{\alpha-1}$, where $\alpha=\alpha_{2}(K), t=t_{\alpha}$, and $s=s(K)$.

\section{Asymptotic behaviour of $M_{0}$ and $m_{0}$ for irrational $r(K)$}

Suppose that $r(K)$ is irrational. Since we have estimates for $M_{0}(x, K)$ and $m_{0}(x, K)$ when $r(K)$ is rational, which could be made even more precise as is shown by the remarks in Section 3, one could suggest that we choose a sequence $K_{i} \rightarrow K$ such that $r\left(K_{i}\right)$ rational, and try to obtain the asymptotic behaviour of $M_{0}(x, K) x^{-\alpha_{1}(K)}$ from the estimates for $M_{0}\left(x, K_{i}\right) x^{-\alpha_{1}\left(K_{i}\right)}$. However, it seems to me that even the best information mentioned in the remarks is far too imprecise for doing this. Therefore we shall consider $M_{0}(x, K)$ and $m_{0}(x, K)$ directly.

We start with the following technical result, which will be proved in Section 9 and which forms the basis for our estimates.

Theorem 4. Let $a, b$ be positive numbers such that $a+b>1$, suppose that $r>1$, and let $C$ be the unique positive zero of the function

$$
P(x)=x^{r}-b x^{r-1}-a .
$$

Then $C>\max \left(1, b, a^{1 / r}\right), P^{\prime}(C)>0$, and the function

$$
S(X)=\sum_{\substack{p, q \geqq 0 \\
p r+q \leqq X}}\left(\begin{array}{c}
p+q \\
q
\end{array}\right) a^{p} b^{q}
$$

satisfies

$$
S(X)=\frac{C^{X+r}}{C P^{\prime}(C) \log C}\left(1+O\left(X^{-\eta}\right)\right)
$$

as $X \rightarrow \infty$, for some positive constant $\eta$. 
Note that $C P^{\prime}(C)=a r+b C^{r-1}$.

Now we can prove the following result.

Theorem 5. If $r=r(K)$ is irrational, then

$$
\lim _{x \rightarrow \infty} M_{0}(x, K) x^{-\alpha_{1}(K)}=\gamma_{1}(K)
$$

and

$$
\lim _{x \rightarrow \infty} m_{0}(x, K) x^{-\alpha_{2}(K)}=\gamma_{2}(K),
$$

where

$$
\gamma_{1}(K)=\alpha^{-1} 2^{1-\alpha}[\log (t+1)]^{\alpha-1}\left[r+2(t+1)^{r-1}\right]^{\alpha-1}, \quad t=t_{\alpha}, \quad \alpha=\alpha_{1}(K),
$$

and

$\gamma_{2}(K)=\alpha^{-1} 2^{1-\alpha}[\log (t+1)]^{\alpha-1}\left[s+2(t+1)^{s-1}\right]^{\alpha-1}, \quad t=t_{\alpha}, \quad \alpha=\alpha_{2}(K), \quad s=s(K)$.

Recall that $(r-1)(s-1)=1$.

6.1. Hayman [3, Theorem 1] proved that the limits (6.4) and (6.5) exist. So it suffices to evaluate them. We have no essentially new proof for the existence of the limits. We consider $M_{0}(x, K)$ only, since the argument for $m_{0}(x, K)$ is similar.

Now we apply Theorem 4 (so we make no further use of the irrationality of $r(K))$ with $r=r(K)$. Take first $a=1, b=2$. Then by [5, Theorem 1], for each $X \geqq 0$, $1+2 S(X)$ is equal to a point $X_{n}$ used in the definition of the function $f \in N_{0}(K)$ (note that the index $p$ in Theorem 4 corresponds to some $p_{k}-1$ in [5, Theorem 1 , (1.3)]). Taking then $a=K, b=2 L$ and denoting the resulting $S(X)$ by $T(X)$, we deduce from [5, Theorem 1, (1.4)] that if $X_{n}=1+2 S(X)$, then $f\left(X_{n}\right)=1+2 K T(X)$. As $X \rightarrow \infty$, we have $X_{n} \rightarrow \infty$. Further, we have $f\left(X_{n}\right)=M_{0}\left(X_{n}, K\right)$ for all $n$ by [5, Theorem 1].

The limit (6.4) is therefore equal to

$$
\lim _{X \rightarrow \infty} 2^{1-\alpha} K T(X) S(X)^{-\alpha}, \quad \alpha=\alpha_{1}(K) .
$$

If $P(X)$ is given by (6.1) with $a=1, b=2$, then $C=t_{\alpha}+1$. To prove this, it suffices to show that $P\left(t_{\alpha}+1\right)=0$, which is a consequence of (4.1) and (4.2) (cf. the argument in subsection 4.1). If $P$ is given by (6.1) with $a=K, b=2 L$, let us denote $P$ by $P_{0}$ and the corresponding $C$ by $C_{0}$. We have $C_{0}=C L$, since $P_{0}(C L)=0$. Namely, $P_{0}(C L)=C^{r} L^{r}-2 L C^{r-1} L^{r-1}-K=0$ since $L^{r}=K$ and since $P(C)=0$.

We conclude from Theorem 4 that the limit (6.4) is equal to

$$
\lim _{X \rightarrow \infty} 2^{1-\alpha} K \frac{(C L)^{X+r} C^{\alpha}(\log C)^{\alpha} P^{\prime}(C)^{\alpha}}{C L(\log C L) P_{0}^{\prime}(C L) C^{\alpha(X+r)}}
$$

Taking into account that $C L=C^{\alpha}$ by (4.2), we obtain (6.4) after some calculations. Theorem 5 is proved. 
Remark. To prove (6.5) we apply Theorem 4 with $a=K^{-1}, b=1+K^{-1}$, so that $a+b=1+2 K^{-1}$. Therefore it is essential to have the assumption $a+b>1$ instead of, for example, $a \geqq 1, b \geqq 1$ in Theorem 4 , even though this makes the proof of Theorem 4 more complicated.

\section{On $\gamma_{1}(K)$ and $\gamma_{2}(K)$}

It may be of some interest to see how $\gamma_{i}(K)$ behaves as $K \rightarrow 1$ or $K \rightarrow \infty$, for $i=1,2$. This gives a better idea of the order of magnitude of $M_{0}(x, K)$ and $m_{0}(x, K)$.

Theorem 6. We have $\gamma_{i}(K) \rightarrow 1$ for $i=1,2$, as $K \rightarrow 1$. As $K \rightarrow \infty$, we have $\gamma_{2}(K) \rightarrow 1$ while

$$
\gamma_{1}(K) \sim B K^{A} / \log K
$$

where $A=(\log 3)^{-1} \log [(3 \log 3) / 2]=0.454676 \ldots$ and

$$
B=(\log 3) \exp \left\{(\log 2)(3 \log 3)^{-1}(\log 4-2 \log \log 3)\right\}=1.41346 \ldots
$$

This should be compared to the estimate [4, Theorem 7]

$$
\log 4 \leqq \liminf _{K \rightarrow \infty} c_{3}(K) \leqq \limsup _{K \rightarrow \infty} c_{3}(K) \leqq \log 9,
$$

where $c_{3}(K)=c_{1}(K) K^{-1} \log \log K$ and

$$
c_{1}(K)=\sup _{x>1} M_{\lrcorner}(x, K) x^{-\alpha_{1}(K)} .
$$

The quantity $c_{1}(K)$ is much larger than $\gamma_{1}(K)$ since $c_{1}(K)$ is affected by $M_{0}(x, K)$ for $x$ close to one.

Consider now $\gamma_{1}(K)$. We use (6.6) together with [4, (5.4)], which reads

$$
\alpha_{1}(K)-1=\log K / \log 3-\log 4 / \log 27+O\left((\log K)^{-1}\right),
$$

and the result in [4, Section 3] that $t_{\alpha} \rightarrow 2$ as $K \rightarrow \infty$, where $\alpha=\alpha_{1}(K)$. This gives

$$
\begin{aligned}
\log \gamma_{1}(K) & =-\log \alpha+(1-\alpha) \log 2+(x-1) \log \log (t+1)+ \\
& +(\alpha-1) \log \left[r+2(t+1)^{r-1}\right],
\end{aligned}
$$

where $\alpha=\alpha_{1}(K), t=t_{\alpha}, r=r(K)$. Hence we see after some calculations that

$$
\log \gamma_{1}(K)=-\log \log K+\log \log 3-(\log K)(\log 2) / \log 3+
$$

$+(\log 2)(\log 4) / \log 27+(\log K)(\log \log (t+1)) / \log 3-(\log 4)(\log \log (t+1)) / \log 27+$

$$
+(\log K)(\log G) / \log 3-(\log 4)(\log G) / \log 27+O(1 / \log K) \text {, }
$$

where $G=r+2(t+1)^{r-1} \rightarrow 3$ as $K \rightarrow \infty$ and $r \rightarrow 1$. Since $(\log K)(r-1)=\log 2+$ $O(1 / \log K)$, we have

$$
\log G=\log 3+(3 \log K)^{-1}(\log 2) \log 9 e+o(1 / \log K)
$$


as $K \rightarrow \infty$. Further, by [4, Lemma 1], we have

$$
\begin{aligned}
\log \log (t+1) & =\log \log L-\log (\alpha-1) \\
& =\log \log 3-(\log 2) /(3 \log K)+O\left((\log K)^{-2}\right),
\end{aligned}
$$

in view of (7.2). Combining these formulas we obtain (7.1).

In fact one can show that

$$
\gamma_{1}(K)=B K^{A}(\log K)^{-1}\left(1+O\left((\log K)^{-1}\right)\right) .
$$

As $K \rightarrow 1$, we have $\alpha_{i}(K) \rightarrow 1$ for $i=1,2$. Thus $\gamma_{i}(K) \rightarrow 1$ for $i=1,2$, by (6.6) and (6.7).

Since $c_{2}(K) \leqq \gamma_{2}(K) \leqq 1$ by (1.4) and since $c_{2}(K) \rightarrow 1$ as $K \rightarrow \infty$ by [4, Theorem 5], we have $\gamma_{2}(K) \rightarrow 1$ as $K \rightarrow \infty$. This proves Theorem 6 .

Remark. One can ask if $\gamma_{1}(K)$ is strictly increasing for $K \geqq 1$. We can show that this is the case at least when $\alpha_{1}(K)>4.54$. Further, we have $\gamma_{2}(K)<1$ for $1<K<\infty$, and $\gamma_{2}(K) \rightarrow 1$ as $K \rightarrow 1$ or $K \rightarrow \infty$. It might be of some interest to determine the minimum value of $\gamma_{2}(K)$.

\section{Asymptotic behaviour of $K$-qs functions}

Let $f$ be $K$-qs. Hayman [3, Theorem 4] showed that if

then

$$
\limsup _{x \rightarrow \infty} f(x) x^{-\alpha_{1}(K)}>0,
$$

and that if

$$
\liminf _{x \rightarrow \infty} f(x) x^{-\alpha_{1}(K)}>0,
$$

then also

$$
\liminf _{x \rightarrow \infty} f(x) x^{-\alpha_{2}(K)}<\infty,
$$

$$
\limsup _{x \rightarrow \infty} f(x) x^{-\alpha_{2}(K)}<\infty .
$$

In fact Hayman's results are more precise, particularly when $r(K)$ is irrational (see [3, Theorems 3, 4]). The above shows that if a $K-q s$ function grows at least sometimes as fast or as slowly as possible, then the function cannot oscillate too much. However, $f$ can oscillate between two powers close to $\alpha_{1}$ and $\alpha_{2}$.

Theorem 7. If $K>1$ and $0<\varepsilon<\left(\alpha_{1}(K)-\alpha_{2}(K)\right) / 2$, we set $\delta_{1}=\left(\alpha_{1}+\alpha_{2}\right) / 2$ and $\delta_{2}=\alpha_{1}-\varepsilon-\delta_{1}$. The odd function $f$ given by

$$
f(x)=\exp \left\{\delta_{1} \log x+\delta_{2} \log x \cos (\eta \log \log (x+e))\right\}
$$

for $x>0$ belongs to $N_{0}(K)$ and satisfies

$$
\limsup _{x \rightarrow \infty} f(x) x^{-\alpha_{1}(K)+\varepsilon} \geqq 1
$$


and

$$
\liminf _{x \rightarrow \infty} f(x) x^{-\alpha_{2}(K)-\varepsilon} \leqq 1
$$

provided that $0<\eta<\eta_{0}$, where $\eta_{0}$ depends on $K$ and $\varepsilon$ only.

Remark. Suppose that $h(x)$ is defined for $x>x_{0}$ and that $h(x) \rightarrow \infty$ as $x \rightarrow \infty$. One can ask if there is $f \in N_{0}(K)$ such that

$$
\limsup _{x \rightarrow \infty} f(x)\left(x^{x_{1}(K)} / h(x)\right)^{-1}>0
$$

and

$$
\liminf _{x \rightarrow \infty} f(x)\left(x^{x_{2}(K)} h(x)\right)^{-1}<\infty .
$$

One could try to find such an odd function $f$ given by

$$
f(x)=\exp \left\{E_{1} \log x+\left[E_{2} \log x-\log h(x)\right] \cos \eta \psi(x)\right\}
$$

for $x>0$, where $E_{1}=\left(\alpha_{1}+\alpha_{2}\right) / 2, E_{2}=\left(\alpha_{1}-\alpha_{2}\right) / 2, \eta$ is a small positive number depending on $K$ and $h$, the function $h$ is assumed to satisfy regularity conditions not essentially affecting its rate of growth, and $\psi(x) \rightarrow \infty$ slowly as $x \rightarrow \infty$, the rate of growth of $\psi$ depending on that of $h$.

It seems to me that such a construction of $f$ might work for some functions $h$ growing more slowly than the powers $h(x)=x^{\varepsilon}$, but the case of an arbitrary $h$ remains open.

8.1. We proceed to prove Theorem 7. To show that the function $f$ given by (8.1) satisfies (1.1), we may assume that $x>0$ and write $x t$ instead of $t$. Then (1.1) is equivalent to

$$
f(x(1+t)) \leqq(K+1) f(x)-K f(x(1-t))
$$

and

$$
f(x(1+t)) \geqq\left(1+K^{-1}\right) f(x)-K^{-1} f(x(1-t)),
$$

which are to be proved for all positive $x$ and $t$. Further, we must show that $f$ is strictly increasing for $x>0$ if $\eta \leqq \eta_{0}$.

For brevity, we write $\psi(x)=\log \log (x+e)$, so that $\psi^{\prime}(x)=[(x+e) \log (x+e)]^{-1}$. We have

$$
\begin{aligned}
x f^{\prime}(x) / f(x) & =\delta_{1}+\delta_{2} \cos \eta \psi(x)-\delta_{2} \eta(\sin \eta \psi(x))(x \log x) \psi^{\prime}(x) \\
& \geqq \delta_{1}-\delta_{2}-\delta_{2} \eta=\alpha_{2}(K)+\varepsilon-\delta_{2} \eta>0
\end{aligned}
$$

if $\eta$ is small enough, so that then $f$ is strictly increasing and defines a homeomorphism of the real axis onto itself. Note that $\log x<\log (x+e)$ for $x \geqq 1$ and that $|x \log x|<$ $1<e \leqq\left|\psi^{\prime}(x)\right|^{-1}$ for $0 \leqq x \leqq 1$. 
8.2. We shall prove (8.6). The proof of (8.7) is similar. In view of (8.1) we can write (8.6) in the form

$$
\begin{gathered}
(1+t)^{\theta_{1}} e^{\theta_{2}} \leqq(K+1)+K(t-1)^{\theta_{3}} e^{\theta_{4}}, \quad t>1, \\
(1+t)^{\theta_{1}} e^{\theta_{2}}+K(1-t)^{\theta_{5}} e^{\theta_{6}} \leqq K+1, \quad 0<t \leqq 1,
\end{gathered}
$$

where

$$
\begin{aligned}
& \theta_{1}=\delta_{1}+\delta_{2} \cos \eta \psi(x t+x), \\
& \theta_{2}=\delta_{2} \log x(\cos \eta \psi(x t+x)-\cos \eta \psi(x)), \\
& \theta_{3}=\delta_{1}+\delta_{2} \cos \eta \psi(x t-x), \\
& \theta_{4}=\delta_{2} \log x(\cos \eta \psi(x t-x)-\cos \eta \psi(x)), \\
& \theta_{5}=\delta_{1}+\delta_{2} \cos \eta \psi(x-x t), \\
& \theta_{6}=\delta_{2} \log x(\cos \eta \psi(x-x t)-\cos \eta \psi(x)) .
\end{aligned}
$$

Note that $\alpha_{2}(K)+\varepsilon \leqq \theta_{1}, \theta_{3}, \theta_{5} \leqq \alpha_{1}(K)-\varepsilon$. Further since for $0<y<z$ we have

we obtain

$$
\begin{aligned}
& |\cos \eta \psi(y)-\cos \eta \psi(z)| \leqq \eta|\psi(y)-\psi(z)| \\
= & \eta \int_{y}^{z} \frac{d u}{(u+e) \log (u+e)} \leqq \frac{\eta(z-y)}{(y+e) \log (y+e)},
\end{aligned}
$$

$$
\begin{aligned}
& \left|\theta_{2}\right| \leqq \eta \delta_{2} x t|\log x|[(x+e) \log (x+e)]^{-1} \leqq B \eta t, \\
& \left|\theta_{4}\right| \leqq \eta \delta_{2} x(t-2)|\log x| \psi^{\prime}(x) \leqq B \eta t \quad \text { if } t \geqq 2, \\
& \left|\theta_{4}\right| \leqq \eta \delta_{2} x(2-t)|\log x| \psi^{\prime}(x t-x) \quad \text { if } \quad 1 \leqq t<2, \\
& \left|\theta_{6}\right| \leqq \eta \delta_{2} x t|\log x| \psi^{\prime}(x-x t),
\end{aligned}
$$

where $B$ depends on $K$ only.

8.3. Recall ([1] or [4, Lemma 1] or Lemma A) that if $\alpha=\alpha_{1}(K)$ then for $1 \leqq \theta \leqq \alpha-\varepsilon$, we have

$$
(1+t)^{\theta} \leqq K+1+K(t-1)^{\theta}-\sigma(\varepsilon, K)\left(1+(t-1)^{\theta}\right)
$$

for $t \geqq 1$, where $\sigma(\varepsilon, K)>0$, and

$$
(1+t)^{\theta}+K(1-t)^{\theta} \leqq K+1-\sigma(K)
$$

for $0<t \leqq 1$, where $\sigma(K)>0$. We deduce from these inequalities and from (8.11) that if $t_{0}=t_{0}(\varepsilon, K)$ is large enough and $\eta=\eta(\varepsilon, K), \tau=\tau(\varepsilon, K)$ and $\omega=\omega(\varepsilon, K)$ are small enough, then

(i) (8.9) holds for $0<t \leqq \tau$ if

$$
\theta_{5} \log (1-t)+\theta_{6} \leqq \omega
$$

for $0<t \leqq \tau$; 
(ii) (8.9) holds for $\tau<t \leqq 1-\tau$ if $\left|\theta_{1}-\theta_{5}\right| \leqq \omega$, since $\left|\theta_{6}\right| \leqq B \eta C(\tau)$, where $C(\tau)$ depends on $\tau$ only;

(iii) (8.9) holds for $1-\tau<t<1$ and hence by continuity for $t=1$ if

$$
\theta_{5} \log (1-t)+\theta_{6} \leqq \omega \log (1-t)
$$

for $1-\tau<t<1$;

(iv) (8.8) holds for $1<t \leqq 1+\tau$;

(v) (8.8) holds for $1+\tau<t<t_{0}$ if $\left|\theta_{1}-\theta_{3}\right| \leqq \omega$ and $\left|\theta_{4}\right| \leqq \omega$; and

(vi) (8.8) holds for $t \geqq t_{0}$ if $t^{\theta_{1}-\theta_{3}} \leqq(K-\omega) e^{\theta_{4}-\theta_{2}}$, i.e.

$$
\delta_{2}(\log t)\left(c_{2}-c_{1}\right) \leqq \log (K-\omega)+\delta_{2}(\log x)\left(c_{1}-c_{2}\right)
$$

for $x>0$ and $t \geqq t_{0}$, where $c_{1}=\cos \eta \psi(x t-x)$ and $c_{2}=\cos \eta \psi(x t+x)$, and $K-\omega>1$.

8.4. It remains to verify that the conditions (i) to (vi) can be satisfied. We shall denote by $B$ or $B_{1}$ any constant depending only on $K$ and $\varepsilon$, not necessarily the same every time.

If $0<t \leqq \tau$, then by (8.11) we have

$$
\begin{aligned}
\theta_{5} \log (1-t)+\theta_{6} & \leqq\left(\alpha_{2}+\varepsilon\right) \log (1-t)+\delta_{2} \eta x t|\log x| \psi^{\prime}(x-x t) \\
& \leqq-\alpha_{2} t+B \eta t<0
\end{aligned}
$$

if $\eta$ is small enough, so that (8.14) holds.

If $\tau<t \leqq 1-\tau$, then

$$
\left|\theta_{1}-\theta_{5}\right| \leqq 2 \eta \delta_{2} x t \psi^{\prime}(x-x t) \leqq B \eta \tau^{-1} \leqq \omega
$$

if $\eta$ is small enough.

If $1-\tau<t<1$, then by (8.11), we have

$$
\theta_{5} \log (1-t)+\theta_{6} \leqq\left(\alpha_{2}+\varepsilon\right) \log (1-t)+\eta \delta_{2} x|\log x| \psi^{\prime}(x-x t),
$$

which does not exceed

$$
\left(\alpha_{2}+\varepsilon\right) \log (1-t)+B \eta \leqq \alpha_{2} \log (1-t) \text { if } 0<x \leqq B_{1},
$$

where $B_{1} \geqq 2$. Suppose that $x>B_{1}$, and choose a positive number $\delta=\delta(K)$ such that $y=\left(\delta_{1}+\delta_{2}\right) /\left(2 \delta_{2}\right)-\delta>1$. If $x \leqq(1-t)^{-\gamma}$, we have

$$
\begin{aligned}
\theta_{5} \log (1-t)+\theta_{6} & \leqq \delta_{1} \log (1-t)-\delta_{2}(\log x) \cos \eta \psi(x)+ \\
& +\delta_{2} \log (x(1-t)) \cos \eta \psi(x-x t) \\
& \leqq\left(\delta_{1}-\gamma \delta_{2}+(1-\gamma) \delta_{2}\right) \log (1-t) \\
& =2 \delta \delta_{2} \log (1-t) \leqq \omega \log (1-t)
\end{aligned}
$$


as required. If $x>(1-t)^{-\gamma}$, then $x(1-t) \geqq x^{1-1 / \gamma} \geqq B_{1}^{1-1 / \gamma}$. Thus

$$
\begin{aligned}
\psi(x)-\psi(x-x t) & =\log \log (x+e)-\log \log (x(1-t)+e) \\
& \leqq-B \log \left[1-\left(\log (1-t)^{-1}\right) /(\log x)\right] \\
& \leqq B\left(\log (1-t)^{-1}\right) / \log x .
\end{aligned}
$$

It follows that $\left|\theta_{6}\right| \leqq B \eta \log (1-t)^{-1}$, so that (8.15) holds in all cases.

8.5. If $1+\tau<t<t_{0}$, then $\left|\theta_{4}\right| \leqq \omega$ by (8.11), and $\left|\theta_{1}-\theta_{3}\right| \leqq B \eta x \psi^{\prime}(x t-x) \leqq \omega$ if $\eta$ is small enough, depending on $\tau$ and $t_{0}$.

Finally if $t \geqq t_{0}$ we assume first that $x\left(t_{0}-1\right) \geqq 1$. We have

$$
\left|c_{1}-c_{2}\right| \leqq 2 \eta x \psi^{\prime}(x t-x) \leqq 2 \eta(t-1)^{-1}(\log (x t-x+e))^{-1},
$$

so that $\left|\delta_{2}(\log x)\left(c_{1}-c_{2}\right)\right| \leqq B \eta$ and $\left|\delta_{2}(\log t)\left(c_{2}-c_{1}\right)\right| \leqq B \eta$. Hence (8.16) holds for some positive $\omega$ if $\eta$ is small enough.

If $x\left(t_{0}-1\right)<1$, we consider separately the possibilities $x t<1$ and $x t \geqq 1$. In each case we have to prove that

$$
(\log x t)\left(c_{2}-c_{1}\right) \leqq \omega_{1}=\delta_{2}^{-1} \log (K-\omega),
$$

which implies (8.16).

If $x t \leqq 1$, then $x<1$ and

$$
\left|(\log x t)\left(c_{2}-c_{1}\right)\right| \leqq B \eta x|\log x t| \leqq B \eta / t \leqq B \eta \leqq \omega_{1} .
$$

If $x t \geqq 1$, then we obtain

$$
\left|(\log x t)\left(c_{2}-c_{1}\right)\right| \leqq 2 \eta x(\log x t)[(x(t-1)+e) \log (x(t-1)+e)]^{-1} \leqq B \eta \leqq \omega_{1},
$$

considering, for example, the cases $x(t-1) \geqq 2,1 \leqq x(t-1) \leqq 2$ and $x(t-1) \leqq 1$. Hence (8.17) and thus (8.16) holds in all cases.

This completes the proof of Theorem 7 .

\section{Proof of Theorem 4}

In this section we prove Theorem 4, stated in Section 6 .

First we note that if $N \geqq 1$, then

$$
1-\frac{B}{N} \leqq N !\left(\frac{N^{N}}{e^{N}}(2 \pi N)^{1 / 2}\right)^{-1} \leqq 1+\frac{B}{N}
$$

where $B=1 / 11$. We shall denote any positive constant by $B$, not necessarily the same every time. We have

$$
\begin{gathered}
\sum_{q \leqq X}\left(\begin{array}{l}
q \\
q
\end{array}\right) b^{q} \leqq B b^{X}=o\left(\frac{C^{X}}{\sqrt{X}}\right), \\
\sum_{p r \leqq X}\left(\begin{array}{l}
p \\
0
\end{array}\right) a^{p} \leqq B a^{X / r}=o\left(\frac{C^{X}}{\sqrt{X}}\right) .
\end{gathered}
$$

Hence we may assume that $p \geqq 1$ and $q \geqq 1$ in (6.2). 
So we have by (9.1),

$$
\left(\begin{array}{c}
p+q \\
q
\end{array}\right) \leqq B \frac{(p+q)^{p+q}}{p^{p} q^{q} \sqrt{2 p}}\left(\frac{1}{p}+\frac{1}{q}\right)^{1 / 2} \leqq B\left(1+\frac{q}{p}\right)^{p}\left(1+\frac{p}{q}\right)^{q},
$$

where instead of $B$ we first have

$$
\left(1+(11(p+q))^{-1}\right)\left(1-(11 p)^{-1}\right)^{-1}\left(1-(11 q)^{-1}\right)^{-1} \leqq 1.265
$$

We write

$$
x=q / X, \quad y=r p /(X-q) .
$$

Since $p, q \geqq 1$ and $p r+q \leqq X$, we have

and

$$
0<\frac{1}{X} \leqq x \leqq 1-\frac{r}{X}<1
$$

$$
0<\frac{r}{X-1} \leqq y \leqq 1 .
$$

We define for $0<x<1,0<y \leqq 1$,

$$
\begin{gathered}
h(x, y)=a^{y(1-x) / r} b^{x}\left(1+\frac{y(1-x)}{r x}\right)^{x}\left(1+\frac{r x}{y(1-x)}\right)^{y(1-x) / r}, \\
h(x)=h(x, 1)=a^{(1-x) / r} b^{x}\left(1+\frac{1-x}{r x}\right)^{x}\left(1+\frac{r x}{1-x}\right)^{(1-x) / r}, \\
T(x, y)=(1-x)\left(\frac{1}{x}+\frac{r}{y(1-x)}\right)^{1 / 2}, \\
T(x)=T(x, 1)=(1-x)\left(\frac{1}{x}+\frac{r}{1-x}\right)^{1 / 2} .
\end{gathered}
$$

Using the values given by (9.5) for $x$ and $y$, we obtain

$$
\begin{gathered}
\left(1+\frac{q}{p}\right)^{p}\left(1+\frac{p}{q}\right)^{q} a^{p} b^{q}=h(x, y)^{X}, \\
\frac{(p+q)^{p+q}}{p^{p} q^{q}}\left(\frac{1}{p}+\frac{1}{q}\right)^{1 / 2} a^{p} b^{q}=h(x, y)^{X} T(x, y) \frac{\sqrt{X}}{X-q} .
\end{gathered}
$$

9.1. We proceed to find some properties of $H(x, y)=\log h(x, y)$ and $H(x)=$ $H(x, 1)$. We have

so that

$$
r H^{\prime \prime}(x)=\frac{(r-1)^{2}}{r x+1-x}-\frac{r}{x}-\frac{1}{1-x}=\frac{-r}{x(1-x)((r-1) x+1)}<0,
$$

$$
r H^{\prime}(x)=\log \frac{b^{r}((r-1)}{a(r x)^{r}}
$$


is strictly decreasing for $0<x<1$, and there is a unique number $x_{0} \in(0,1)$ such that $H^{\prime}\left(x_{0}\right)=0$. Then $H^{\prime \prime}\left(x_{0}\right)<0$, and $H(x)$ has its unique global maximum at $x=x_{0}$. Further, $H(x)$ is strictly increasing for $0<x \leqq x_{0}$ and strictly decreasing for $x_{0} \leqq x<1$.

Using the fact that $H^{\prime}\left(x_{0}\right)=0$, we see after a lengthy but routine calculation that $P\left(h\left(x_{0}\right)\right)=0\left(h\left(x_{0}\right)=e^{H\left(x_{0}\right)}\right)$. Hence $h\left(x_{0}\right)=C$ and $H\left(x_{0}\right)>0$. A calculation also shows that the number $\gamma$ defined by

satisfies

$$
\gamma=\left(\frac{1}{x_{0}}+\frac{r}{1-x_{0}}\right)^{1 / 2}\left\{\left(\log \left(1+\frac{r x_{0}}{1-x_{0}}\right)\right)\left(-H^{\prime \prime}\left(x_{0}\right)\right)^{1 / 2}\right\}^{-1}
$$

and that

$$
\gamma=C^{r-1} /\left(P^{\prime}(C) \log C\right),
$$

We omit the details.

9.2. Our next aim is to show that $H\left(x_{0}\right)=H\left(x_{0}, 1\right)$ is the unique maximum of $H(x, y)$ for $0<x<1,0<y \leqq 1$. We have

$$
r \frac{\partial}{\partial y} H(x, y)=(1-x) \log \left(a\left(1+\frac{r x}{y(1-x)}\right)\right) .
$$

For a fixed $x$, either this is positive for $0<x<1$, in which case $H(x, y)$ is strictly increasing, or there is $y_{0}$ such that $\partial_{y} H(x, y)>0$ for $0<y<y_{0}$ and $\partial_{y} H(x, y)<0$ for $y_{0}<y<1$. In the former case $H(x, y)<H(x)<H\left(x_{0}\right)$ for $0<y<1$ and $x \neq x_{0}$, and $H\left(x_{0}, y\right)<H\left(x_{0}\right)$ for $0<y<1$. In the latter case we have $0<a<1$ and $x<$ $x_{0}-\varepsilon_{1}$ for some $\varepsilon_{1}$. Namely, if $x \geqq x_{0}-\varepsilon_{1}$ and $0<y \leqq 1$, then since $x /(1-x)$ is an increasing function of $x$, we have

$$
\begin{aligned}
\log a\left(1+\frac{r x}{y(1-x)}\right) & \geqq \log a\left(1+\frac{r\left(x_{0}-\varepsilon_{1}\right)}{y\left(1-x_{0}+\varepsilon_{1}\right)}\right) \\
& \geqq \log a\left(1+\frac{r\left(x_{0}-\varepsilon_{1}\right)}{1-x_{0}+\varepsilon_{1}}\right)>0
\end{aligned}
$$

by (9.11) if $\varepsilon_{1}$ is small enough.

If there are any $x, y$ with $\partial_{y} H(x, y)=0$, then clearly there is $x_{1} \in\left(0, x_{0}-\varepsilon_{1}\right)$ with the following property. For every $x \in\left(0, x_{1}\right)$ there is $y=y_{0}(x) \in(0,1)$ such that $\partial_{y} H(x, y)=0$ i.e. $a\left(1+r x /\left[y_{0}(x)(1-x)\right]\right)=1$, while for $x \in\left(x_{1}, 1\right), H(x, y)$ is a strictly increasing function of $y$. Suppose that this is the case. Since $h(0, y)=1<$ $h\left(x_{0}\right)$, it suffices to show that

$$
\sup _{0<x \leq x_{1}} H\left(x, y_{0}(x)\right)<H\left(x_{0}\right) .
$$

As we must have $y_{0}\left(x_{1}\right)=1$, we have $H\left(x, y_{0}(x)\right) \leqq H\left(x_{0}\right)-\varepsilon_{2}$ by continuity for some positive $\varepsilon_{2}$ and for $x_{1}-\varepsilon_{2} \leqq x \leqq x_{1}$. If $H\left(x, y_{0}(x)\right)>1$ for any $x \leqq x_{1}-\varepsilon_{2}$, then 
$H\left(x, y_{0}(x)\right)$ attains a maximum on $\left(0, x_{1}-\varepsilon_{2}\right]$. So it suffices to prove that $H\left(x, y_{0}(x)\right)<H\left(x_{0}\right)$ for these $x$, i.e. that $D=e^{H\left(x, y_{0}(x)\right)}<C$. Using the fact that $\partial_{y} H(x, y)=0$ for $y=y_{0}(x)$, we obtain

$$
1<D=\left(\frac{b}{1-a}\right)^{x}<\left(\frac{b}{1-a}\right)^{x_{1}}=D_{0}<\frac{b}{1-a}
$$

since $b>1-a$. Further since $y_{0}\left(x_{1}\right)=1$, we have

$$
x_{1}=\frac{1-a}{a r+1-a} .
$$

As $P\left(D_{0}\right) \leqq P(C)=0$ implies that $D_{0} \leqq C$, we shall show that for any fixed $a \in(0,1)$ and $r>1$, the expression $P\left(D_{0}\right)$ is $\leqq 0$ for any $b>1-a$, where $D_{0}$ and $x_{1}$ are as above. This reads

$$
h_{1}(b)=-A_{1} b^{e_{1}}+a+A_{2} b^{e_{1}+e_{2}} \geqq 0,
$$

where $A_{1}=(1-a)^{-r x_{1}}, A_{2}=(1-a)^{(1-r) x_{1}}, e_{1}=r x_{1}, e_{2}=1-x_{1}>0$, and $h_{1}(1-a)=0$, while $h_{1}^{\prime}(b)>0$ for $b>1-a$. Thus $h_{1}(b)>0$ for $b>1-a$. This proves that $H\left(x_{0}\right)$ is the unique maximum of $H(x, y)$.

9.3. Now we can estimate $S(X)$. Suppose that $0<\delta<1 / 2, \varepsilon>0,0<x_{0}-\varepsilon<$ $x_{0}+\varepsilon<1$, and set

$$
V(\delta, \varepsilon)=\left\{(p, q)|p, q \geqq 1, p r+q \leqq X,| x-x_{0} \mid \geqq \varepsilon \text { or } y \leqq 1-\delta\right\}
$$

where $x$ and $y$ are given by (9.5). With

we have by (9.4) and (9.6),

$$
U(p, q)=\left(\begin{array}{c}
p+q \\
q
\end{array}\right) a^{p} b^{q}
$$

$$
\sum_{V(\delta, \varepsilon)} U(p, q) \leqq B \sum_{V(\delta, \varepsilon)} h(x, y)^{X} \leqq B X^{2}(\eta C)^{X}=o\left(\frac{C^{X}}{\sqrt{X}}\right),
$$

where $\eta=\eta(\delta, \varepsilon, r, a, b) \in(0,1)$ is such that $h(x, y) \leqq \eta C$ if $\left|x-x_{0}\right| \geqq \varepsilon$ or $y \leqq 1-\delta$. Note that there are at most $B X^{2}$ terms in the sum.

We set

$$
W=W(\delta, \varepsilon)=\left\{(p, q)|p, q \geqq 1, p r+q \leqq X,| x-x_{0} \mid<\varepsilon \text { and } y>1-\delta\right\} .
$$

If $(p, q) \in W$, then with

we have

$$
R(p, q)=\frac{(p+q)^{p+q}}{p^{p} q^{q} \sqrt{2 \pi}}\left(\frac{1}{p}+\frac{1}{q}\right)^{1 / 2},
$$

$$
\left|\left(\begin{array}{c}
p+q \\
q
\end{array}\right)-R(p, q)\right| \leqq B R(p, q) X^{-1} .
$$

Hence it suffices to prove the asymptotic formula (6.3) for

$$
F(X)=\sum_{W} R(p, q) a^{p} b^{q}
$$


instead of $S(X)$. That together with our earlier estimates (9.2), (9.3), (9.13) and (9.14) then proves Theorem 4.

By (9.7) we have

$$
F(X)=\frac{X \sqrt{X}}{r \sqrt{2 \pi}} \sum_{W} e^{X H(x, y)} T(x, y) \frac{1}{X} \frac{r}{X-q},
$$

where $x, y$ are given by (9.5). Here $q$ runs from $X\left(x_{0}-\varepsilon\right)$ to $X\left(x_{0}+\varepsilon\right)$ and for each $q$, $p$ runs from $(1-\delta)(X-q) / r$ to $(X-q) / r$. Hence any successive values of $x$ and $y$, say $x_{1}$ and $x_{2}$, or $y_{1}$ and $y_{2}$, satisfy

$$
\left|x_{1}-x_{2}\right|=1 / X, \quad\left|y_{1}-y_{2}\right| \leqq B / X,
$$

where $B$ depends on $r$ and $x_{0}$. Using this and the monotonicity properties of $H(x, y)$, one can show that

$$
\left|F(X)-\frac{X^{3 / 2}}{r \sqrt{2 \pi}} I(X)\right| \leqq \frac{B C^{X}}{\sqrt{X}},
$$

where

$$
I(X)=\int_{x_{0}-\varepsilon}^{x_{0}+\varepsilon} \int_{1-\delta}^{1} e^{X H(x, y)} T(x, y) d x d y,
$$

provided that $\delta$ and $\varepsilon$ are small enough and $X$ is large enough. We omit the details.

9.4. It remains to estimate $I(X)$ for small but fixed $\delta$ and $\varepsilon$ as $X \rightarrow \infty$. In particular, we make sure that $x_{1}<x_{0}-\varepsilon$, if there exists $x_{1}$ as above. We note that

$$
0<H(x)-H(x, y)=(1-y) \partial_{y} H(x, \xi)
$$

for some $\xi \in(y, 1)$. If $y \leqq 1-B X^{-1} \log X$ for a suitable $B$ then

$$
\begin{aligned}
H(x, y) & \leqq H\left(x_{0}\right)-(1-y) \min _{y \geqq \xi \leqq 1} \partial_{y} H(x, \xi) \\
& =\log C-(1-y)(1-x) r^{-1} \log a\left(1+\frac{r x}{1-x}\right) \\
& \leqq \log C-5 X^{-1} \log X
\end{aligned}
$$

and $\exp X H(x, y) \leqq C^{X} X^{-5}$. Hence

$$
\int_{x_{0}-\varepsilon}^{x_{0}+\varepsilon} \int_{1-\delta}^{1-B X^{-1} \log X} T(x, y) e^{X H(x, y)} d x d y \leqq B C^{X} X^{-5} .
$$

Consider next

$$
E(x, y)=H(x)-H(x, y)-(1-y) \partial_{y} H(x, 1)
$$

for $\left|x-x_{0}\right| \leqq \varepsilon, 1-B X^{-1} \log X \leqq y \leqq 1$. Let $x$ be fixed. We have $E(x, 1)=0$. Hence $-E(x, y)=(1-y) \partial_{y} E(x, \xi)$ for some $\xi \in(y, 1)$. We have

$$
\partial_{y} E(x, y)=-\partial_{y} H(x, y)+\partial_{y} H(x, 1)=(1-y) \partial_{y y} H\left(x, \xi_{1}\right)
$$


for some $\xi_{1} \in(y, 1)$. So

$$
-E(x, y)=(1-y)(1-\xi) \partial_{y y} H\left(x, \xi_{1}\right)
$$

where $y \leqq \xi \leqq \xi_{1} \leqq 1$. We have

$$
\partial_{y y} H(x, y)=\frac{-x(1-x)}{y(y(1-x)+r x)}<0,
$$

and $\left|\partial_{y y} H(x, y)\right| \leqq B$ for $\left|x-x_{0}\right| \leqq \varepsilon, 1-\delta \leqq y \leqq 1$. Thus

$$
0 \leqq E(x, y) \leqq B(1-y)^{2} \leqq B X^{-2}(\log X)^{2} .
$$

It follows that

$$
\begin{aligned}
e^{X H(x, y)} & =e^{X H(x)} e^{-X(1-y) \delta_{y} H(x, 1)} e^{-X E(x, y)} \\
& =e^{X H(x)} \exp \left\{-X(1-y)(1-x) r^{-1} \log \left(1+\frac{r x}{1-x}\right)\right\}\left(1+E_{1}\right) \\
& =e^{X H(x)}(\exp g(x, y))\left(1+E_{1}\right),
\end{aligned}
$$

say, where

and so

$$
E_{1}=E_{1}(x, y)=1-\exp (-X E(x, y))
$$

Hence

$$
\left|E_{1}\right| \leqq B X^{-1}(\log X)^{2} .
$$

$$
\begin{gathered}
\int_{x_{0}-\varepsilon}^{x_{0}+\varepsilon} \int_{1-B X^{-1} \log X}^{1} e^{X H(x, y)} T(x, y) d x d y \\
=\iint e^{X H(x)} T(x, y) e^{g(x, y)} d x d y\left(1+O(1) X^{-1}(\log X)^{2}\right) .
\end{gathered}
$$

A similar argument shows that

$$
T(x, y)=T(x, 1)+O(1) X^{-1} \log X,
$$

so that we can replace $T(x, y)$ by $T(x)$ in the above integral. The resulting integral depends on $y$ only through $1-y$ in the exponent, so that integrating with respect to $y$ we obtain

$$
\int_{x_{0}-\varepsilon}^{x_{0}+\varepsilon} \frac{e^{X H(x)}}{X(1-x) \log (1+r x /(1-x))}\left(1-\exp \left\{-B(\log X) B_{1}(x)\right\}\right),
$$

where $B_{1}(x)=(1-x) \log (1+r x /(1-x))$. The second term in brackets is $O\left(X^{-\eta}\right)$ for some positive $\eta<1$. To deal with the first term we use the following standard result (see e.g. [2, Theorem 2, p. 19]).

Lemma B. Let $H(x)$ and $q(x)$ be analytic functions of $x$, regular on $(c, d)$ and continuous on $[c, d]$. Let $H$ be real and suppose that $H$ attains its maximum at $x=x_{0} \in$ $(c, d)$ only, while $H^{\prime \prime}\left(x_{0}\right)<0$. Then as $X \rightarrow \infty$, we have

$$
\int_{c}^{d} e^{X H(x)} q(x) d x=e^{X H\left(x_{0}\right)} q\left(x_{0}\right)\left(\frac{2 \pi}{X\left|H^{\prime \prime}\left(x_{0}\right)\right|}\right)^{1 / 2}\left(1+\frac{O(1)}{X}\right) .
$$


Applying Lemma $B$ to our present $H(x)$ and to

$$
q(x)=r T(x)[X(1-x) \log (1+r x /(1-x))]^{-1},
$$

as we may in view of what we have proved about $H(x)$, we see that the integral (9.17) is equal to

$$
\left(\frac{2 \pi}{X}\right)^{1 / 2} \frac{r e^{X H\left(x_{0}\right)} T\left(x_{0}\right)\left(-H^{\prime \prime}\left(x_{0}\right)\right)^{-1 / 2}}{X\left(1-x_{0}\right) \log \left(1+r x_{0} /\left(1-x_{0}\right)\right)}\left(1+O\left(X^{-1}\right)\right) .
$$

Using this, the definition of $T(x),(9.16)$, and our earlier estimates we get

$$
S(X)=\gamma C^{X}\left(1+O\left(X^{-\eta}\right)\right) .
$$

In view of $(9.10)$, this proves Theorem 4.

\section{References}

[1] Beurling, A., and L. Ahlfors: The boundary correspondence under quasiconformal mappings. - Acta Math. 96, 1956, 125-142.

[2] Evgrafov, M. A.: Asymptotic estimates and entire functions. - Gordon and Breach, Science Publishers, Inc., New York, 1961.

[3] HaYman, W. K.: The asymptotic behaviour of K. q. s. functions. - Mathematical StructuresComputational Mathematics-Mathematical Modelling, 2, Papers dedicated to Academician L. Iliev's 70th Anniversary, Publishing House of the Bulgarian Academy of Sciences, Sofia, 1984, 198-207.

[4] Hayman, W. K., and A. Hinkkanen: Distortion estimates for quasisymmetric functions. - Ann. Univ. Mariae Curie-Skłodowska Sect. A. 36-37, 1982-83 (published 1985), 5167.

[5] HinkKanen, A.: Quasisymmetric functions of extremal growth. - Ann. Acad. Sci. Fenn. Ser. A I Math. 11, 1986, 63-75.

[6] KeLingos, J. A.: Boundary correspondence under quasiconformal mappings. - Michigan Math. J. 13, 1966, 235-249, 63-75.

University of Michigan

Department of Mathematics

Ann Arbor, Michigan 48109

USA

Received 23 May 1985 